\title{
On the minimal energy state of a mixture of charged classical and quantum fluids
}

\author{
Nirmalendu Chaudhuri* \\ Department of Mathematics \\ Indian Institute of Science \\ Bangalore - 560 012, India
}

\author{
and Andreas Unterreiter ${ }^{\dagger}$ \\ Universität Kaiserslautern \\ Erwin-Schrödinger Straße \\ D - 67663 Kaiserslautern, Germany
}

August 16, 2000

\begin{abstract}
The paper concerns the equilibrium state of ultra small semiconductor devices. Due to the quantum drift diffusion model, electrons and holes behave as a mixture of charged quantum fluids. Typically the involved scaled Planck's constants of holes, $\xi$, is significantly smaller than the scaled Planck's constant of electrons. By setting formally $\xi=0$ a well-posed differential-algebraic system arises. Existence and uniqueness of an equilibrium solution is proved. A rigorous asymptotic analysis shows that this equilibrium solution is the limit (in a rather strong sense) of quantum systems as $\xi \rightarrow 0$. In particular the ground state energies of the quantum systems converge to the ground state energy of the differential-algebraic system as $\xi \rightarrow 0$.
\end{abstract}

Key words. thermal equilibrium state; charged fluids; mixture of quantum fluids and classical fluids; semi-classical limits.

AMS(MOS) subject classification. 35B45, 35B65, 35S20, 35Q35, 35Q40, 49S05, 76Y05.

${ }^{*}$ N. C. thanks DAAD for providing the financial support through the exchange programme, which made this research work possible, and the Fachbereich Mathematik, for providing all the necessary facilities. N. C. also profusely thanks Prof. H.Neunzert and Prof. M.Pandit for their encouragement.

${ }^{\dagger}$ A. U. acknowledges support from the TMR network "Asymptotic Methods in Kinetic Theory", No. ERBFRMXCT 970157. 


\section{Introduction}

Quantum Drift Diffusion models (QDDs) $[3,6]$ give a rather accurate account of the macroscopic behavior of ultra small semiconductor devices in terms of particle densities, current densities and electric fields. In particular, QDDs allow for the modelization of several quantum effects on macroscopic scales [7]. These features of QDDs encourage investigations of several scaling limits which arise in practical situations $[8,9]$.

The scaling limits investigated so far are settled on the assumption that both charge carriers - electrons and holes - exhibit quantum properties. However, the effective masses of electrons and holes may differ significantly [2]. As a consequence, the scaled Planck's constants of electrons and holes are in several situations of different orders of magnitude.

In particular the case "scaled Planck's constant for holes" « "scaled Planck's constant for electrons" is of distinguished importance [2]. In this situation one has to expect that holes behave as a classical charged fluid while electrons exhibit quantum phenomena.

It is the aim of this paper to discuss the thermal equilibrium state of a bipolar charged quantum fluid when the scaled Planck's constant of one of the two components is significantly smaller than the other.

To fix ideas let us give the precise definition of the problem.

We consider the QDD of a bipolar charged quantum fluid confined to $\Omega$ where we assume

(A1) $\Omega \subset \mathbb{R}^{d}, d=1,2$ or $d=3$ is a non void, bounded domain.

$H^{1}(\Omega)$ is continuously embedded in $L^{6}(\Omega)$ and $H^{1}(\Omega)$ is compactly embedded in $L^{4}(\Omega)$.

Remark 1. Since $d \leq 3$, the embeddings of $H^{1}(\Omega)$ hold, e.g., for domains $\Omega$ with Lipschitz boundary [1].

The term "bipolar" refers to two particle types, namely negatively charged "electrons" and positively charged "holes". The particle density of electrons is $n_{\xi}=$ $n_{\xi}(x) \geq 0$ and the particle density of holes is $p_{\xi}=p_{\xi}(x) \geq 0$, where $x$ ranges in $\Omega$.

The thermal equilibrium state of the system is governed by the semi linear elliptic system $[2,8]$

$$
\begin{aligned}
\varepsilon^{2} \Delta \sqrt{n_{\xi}} & =\sqrt{n_{\xi}}\left(\log \left(n_{\xi}\right)+V_{\xi}+\alpha_{\xi}\right) \\
\varepsilon^{2} \xi \Delta \sqrt{p_{\xi}} & =\sqrt{p_{\xi}}\left(\log \left(p_{\xi}\right)-V_{\xi}+\beta_{\xi}\right) \\
-\lambda \Delta V_{\xi} & =n_{\xi}-p_{\xi}-C,
\end{aligned}
$$


where $V_{\xi}=V_{\xi}(x)$ is the electrostatic potential, the real numbers $\varepsilon>0$ and $\varepsilon \xi>0$ are the scaled Planck's constants of electrons and holes, respectively, $\lambda>0$ is the scaled minimal Debye length and the function

(A2) $C \in L^{\infty}(\Omega)$

represents a fixed background ion distribution.

System (1)-(3) is supplied with the constraints

$$
\begin{aligned}
\int_{\Omega} n_{\xi}(x) d x & =N>0 \\
\int_{\Omega} p_{\xi}(x) d x & =P>0 \\
\int_{\Omega} V_{\xi}(x) d x & =0
\end{aligned}
$$

and

$V_{\xi}$ satisfies homogeneous Neumann boundary conditions,

i.e. we have to impose global charge neutrality

(A3) $N-P=\int_{\Omega} C(x) d x$.

In (1) - (7) the functions $n_{\xi}, p_{\xi}, V_{\xi}$ and the real numbers $\alpha_{\xi}, \beta_{\xi}$ are unknown. Since our main conclusions do not depend on the particular choice of $\varepsilon, \lambda$ we set

$$
\varepsilon=\lambda=1
$$

henceforth.

Following [8] the analysis of (1)-(7) proceeds by introducing the corresponding energy functional $J_{\xi}$ which is constructed in several steps.

$J_{\xi}$ is the sum of a $\xi$-dependent quantum energy term and $\xi$-independent enthalpy and electrostatic energy terms.

In the general context considered here we have to take a closer look at the electrostatic energy $E_{\infty}$. We introduce the sets

$$
\mathcal{C}_{0}:=\left\{\phi \in H^{1}(\Omega): \int_{\Omega} \phi d x=0\right\}
$$

and

$$
\begin{aligned}
& \mathcal{C}_{e l}:=\left\{f \in L^{1}(\Omega):\right. \\
&\left.\quad \exists V \in \mathcal{C}_{0}:\left(\forall \phi \in \mathcal{C}_{0} \cap L^{\infty}(\Omega): \int_{\Omega} \nabla V \cdot \nabla \phi d x=\int_{\Omega} f \phi d x\right)\right\} .
\end{aligned}
$$


It is easy to see: for each $f \in \mathcal{C}_{e l}$ there is exactly one $V \in \mathcal{C}_{0}$ such that $\int_{\Omega} \nabla V$. $\nabla \phi d x=\int_{\Omega} f \phi d x$ for all $\phi \in \mathcal{C}_{0} \cap L^{\infty}(\Omega)$.

We denote this $V \in \mathcal{C}_{0}$ by $V[f]$ henceforth.

Remark 2. a) If $f \in \mathcal{C}_{\text {el }}$, then $V=V[f]$ is the unique weak solution of

$$
-\Delta V=f-[f], \quad V \in \mathcal{C}_{0}
$$

where

$$
[f]:=\frac{1}{\operatorname{meas}(\Omega)} \int_{\Omega} f d x
$$

subject to homogeneous Neumann boundary conditions, i.e.

$$
\int_{\Omega} \nabla V \cdot \nabla \phi d x=\int_{\Omega}(f-[f]) \phi d x
$$

for all $\phi \in H^{1}(\Omega) \cap L^{\infty}(\Omega)$.

b) In particular we have $L^{2}(\Omega) \subset \mathcal{C}_{\text {el }}$.

c) By a density argument one easily verifies

$$
\forall h \in L^{2}(\Omega), \forall f \in \mathcal{C}_{e l}: \quad \int_{\Omega} \nabla V[h] \cdot \nabla V[f] d x=\int_{\Omega} h V[f] d x .
$$

d) The set $\mathcal{C}_{e l}$ is not closed with respect to weak convergence in $L^{1}(\Omega)$.

We assume

(A4) There exists a $K_{\infty}>0$ only depending on $\Omega$ such that

$$
\|V[f]\|_{L^{\infty}(\Omega)} \leq K_{\infty}\|f\|_{L^{2}(\Omega)}, \quad \text { for all } f \in L^{2}(\Omega) \text { with } \int_{\Omega} f d x=0 .
$$

Remark 3. Assumption (A4) is a requirement on the smoothness of $\partial \Omega$. For instance it is well known, see e.g. [4], that for $\partial \Omega \in C^{\infty}$ the estimate

$$
\|V[f]\|_{H^{2}(\Omega)} \leq K\|f\|_{L^{2}(\Omega)}
$$

holds. This estimate implies for domains $\Omega$ with Lipschitz boundary in dimensions $d \leq 3$ assumption (A4), because in this situation the embedding $H^{2}(\Omega) \rightarrow C_{B}(\Omega)$ is continuous [1].

Now we introduce the electrostatic energy functional

$$
E_{\infty}: L^{1}(\Omega) \rightarrow \mathbb{R}_{0}^{+} \cup\{\infty\}, \quad E_{\infty}(f)= \begin{cases}\frac{1}{2} \int|\nabla V[f]|^{2} d x & f \in \mathcal{C}_{e l} \\ \infty & , f \notin \mathcal{C}_{e l}\end{cases}
$$


and the energy functional corresponding to (1)-(7),

$$
\begin{aligned}
J_{\xi}: \Gamma \rightarrow \mathbb{R}, \quad & J_{\xi}(n, p)=\int_{\Omega}|\nabla \sqrt{n}|^{2} d x+\xi \int_{\Omega}|\nabla \sqrt{p}|^{2} d x \\
& +\int_{\Omega} n(\log (n)-1) d x+\int_{\Omega} p(\log (p)-1) d x+E_{\infty}(n-p-C),
\end{aligned}
$$

where

$$
\begin{aligned}
\Gamma:=\left\{(n, p) \in L^{1}(\Omega) \times L^{1}(\Omega): n, p \geq 0, \sqrt{n}, \sqrt{p} \in H^{1}(\Omega),\right. & \\
& \left.\int_{\Omega} n d x=N, \int_{\Omega} p d x=P\right\} .
\end{aligned}
$$

Remark 4. If $(n, p) \in \Gamma$, then $\sqrt{n}, \sqrt{p} \in H^{1}(\Omega)$. Hence $n, p \in L^{2}(\Omega)$ and therefore $\Gamma \subset \mathcal{C}_{\text {el }} \times \mathcal{C}_{\text {el }}$, i.e. $E_{\infty}(n-p-C)<\infty$. Furthermore, due to sub quadratic growth of $t \log (t)$ as $t \rightarrow \infty$, we have $n \log (n), p \log (p) \in L^{1}(\Omega)$. Hence $J_{\xi}(n, p)<\infty$ for all $\xi \in(0, \infty)$.

Following [8] we have

Theorem 1. [8] Assume (A1)-(A4). Then $J_{\xi}$ has for each $\xi \in(0, \infty)$ exactly one minimizer $\left(n_{\xi}, p_{\xi}\right) \in \Gamma$, i.e.

$$
J_{\xi}\left(n_{\xi}, p_{\xi}\right)=\min _{\Gamma} J_{\xi}
$$

Furthermore, for all $\xi \in(0, \infty)$,

1. there are real numbers $\alpha_{\xi}, \beta_{\xi}$ such that the quintuple $\left(n_{\xi}, p_{\xi}, V_{\xi}, \alpha_{\xi}, \beta_{\xi}\right)$ where $V_{\xi}=V\left[n_{\xi}-p_{\xi}-C\right]-$ is a solution of $(1)-(7)$,

2. there are real numbers $m_{\xi}, M_{\xi} \in(0, \infty)$ such that

$$
m_{\xi} \leq n_{\xi}, p_{\xi} \leq M_{\xi}, \quad\left|V_{\xi}\right| \leq M_{\xi}
$$

3. $\sqrt{n_{\xi}}, \sqrt{p_{\xi}}$ satisfy homogeneous Neumann boundary conditions, i.e.

$$
\sqrt{n_{\xi}}=-V\left[\sqrt{n_{\xi}}\left(\log \left(n_{\xi}\right)+V_{\xi}+\alpha_{\xi}\right)\right], \sqrt{p_{\xi}}=-V\left[\sqrt{p_{\xi}}\left(\log \left(p_{\xi}\right)-V_{\xi}+\beta_{\xi}\right)\right] .
$$

Remark 5. a) The real numbers $\alpha_{\xi}, \beta_{\xi}$ are the Lagrange multipliers of the minimization problem $J_{\xi} \rightarrow$ Min on $\Gamma$.

b) As a consequence of 1 . and of 3. we have for each $\xi \in(0, \infty)$,

$$
\int_{\Omega} \sqrt{n_{\xi}}\left(\log \left(n_{\xi}\right)+V_{\xi}+\alpha_{\xi}\right) d x=\int_{\Omega} \sqrt{p_{\xi}}\left(\log \left(p_{\xi}\right)-V_{\xi}+\beta_{\xi}\right) d x=0 .
$$


In the sequel we shall be concerned with the limit $\xi \rightarrow 0$, in particular with the following questions.

a) Setting $\xi=0$ in (1)-(7) we obtain

$$
\begin{aligned}
\Delta \sqrt{n_{0}} & =\sqrt{n_{0}}\left(\log \left(n_{0}\right)+V_{0}+\alpha_{0}\right) \\
0 & =\log \left(p_{0}\right)-V_{0}+\beta_{0} \\
-\Delta V_{0} & =n_{0}-p_{0}-C, \\
\int_{\Omega} n_{0}(x) d x & =N>0, \\
\int_{\Omega} p_{0}(x) d x & =P>0, \\
\int_{\Omega} V_{0}(x) d x & =0,
\end{aligned}
$$

and

$V_{0}$ satisfies homogeneous Neumann boundary conditions.

The question is: Does this differential-algebraic system has an equilibrium solution $\left(n_{0}, p_{0}, V_{0}, \alpha_{0}, \beta_{0}\right)$, i.e. is there a minimizer $\left(n_{0}, p_{0}\right)$ of the energy

$$
\begin{aligned}
& J_{0}: \Gamma_{0} \rightarrow \mathbb{R}_{0}^{+} \cup\{\infty\}, \\
& J_{0}(n, p)=\int_{\Omega}|\nabla \sqrt{n}|^{2} d x+\int_{\Omega} n(\log (n)-1) d x+\int_{\Omega} p(\log (p)-1) d x \\
& +E_{\infty}(n-p-C),
\end{aligned}
$$

where

$$
\begin{aligned}
\Gamma_{0}:=\left\{(n, p) \in L^{1}(\Omega) \times \mathcal{C}_{e l}: n, p \geq 0, \sqrt{n} \in H^{1}(\Omega),\right. \\
\left.\qquad \int_{\Omega} n d x=N, \int_{\Omega} p d x=P\right\},
\end{aligned}
$$

such that $\left(n_{0}, p_{0}, V\left[n_{0}-p_{0}-C\right], \alpha_{0}, \beta_{0}\right)$ - where the real numbers $\alpha_{0}, \beta_{0}$ are the Lagrange multipliers of minimizing $J_{0}$ in $\Gamma_{0}$ - is a solution of (8)-(14) ?

b) In which sense - if at all - do we have

$$
n_{\xi} \rightarrow n_{0}, p_{\xi} \rightarrow p_{0}, V\left[n_{\xi}-p_{\xi}-C\right] \rightarrow V\left[n_{0}-p_{0}-C\right], \alpha_{\xi} \rightarrow \alpha_{0}, \beta_{\xi} \rightarrow \beta_{0},
$$

as $\xi \rightarrow 0 ?$ 
c) Do we have $\lim _{\xi \rightarrow 0} \min _{\Gamma} J_{\xi}=\min _{\Gamma_{0}} J_{0} ?$

The paper is organized as follows.

In section 2 we investigate system (8)-(14). The main result is theorem 2 which is an analogon to theorem 1. In particular we prove existence and uniqueness of an equilibrium solution minimizing $J_{0}$ in $\Gamma_{0}$. In contrast to the proof of theorem 1 the derivation of a priori estimates for $p_{0}$ is a bit delicate. Since $p_{0}$ satisfies an algebraic equation no maximum principle can be applied. Instead, one has to use monotonicity operators for a semi linear elliptic operator acting on the potential $V_{0}$. As a consequence of theorem 2, question a) has an affirmative answer.

Section 3 addresses to the convergence of the minimizers of $J_{\xi}$ to the minimizer of $J_{0}$ as $\xi \rightarrow 0$. In theorem 3 the corresponding results are collected. For the particle densities uniform pointwise bounds away from zero and away from $\infty$ are proved. This result extends theorem 1 where the corresponding constants are $\xi$-dependent. Questions b) and c) are given affirmative answers. Furthermore the convergence of and of $\min _{\Gamma} J_{\xi}$ to $\min _{\Gamma_{0}} J_{0}$ as $\xi \rightarrow 0$ is proved. This result is rather important for physical reasons: The ground state energy of the approximating quantum/classical system is the limiting value of the ground state energies of quantum/quantum systems as the scaled Planck's constant of the respective particle type tends to zero.

The proof of theorem 2 is deferred to the Appendix.

\section{Variational Analysis of $J_{0}$}

In this section we are concerned with the minimization of $J_{0}$ in $\Gamma_{0}$ and with the derivation of the corresponding Euler Lagrange equations.

The main result is

Theorem 2. Assume (A1)-(A4). Then $J_{0}$ has exactly one minimizer $\left(n_{0}, p_{0}\right)$ in $\Gamma_{0}$, i.e.

$$
J_{0}\left(n_{0}, p_{0}\right)=\min _{\Gamma_{0}} J_{0}
$$

Furthermore,

1. there are real numbers $\alpha_{0}, \beta_{0}$ such that the quintuple $\left(n_{0}, p_{0}, V_{0}, \alpha_{0}, \beta_{0}\right)$ where $V_{0}=V\left[n_{0}-p_{0}-C\right]-i s$ a solution of (8)-(14),

2. there are real numbers $m_{0}, M_{0} \in(0, \infty)$ such that

$$
m_{0} \leq n_{0}, p_{0} \leq M_{0}, \quad\left|V_{0}\right| \leq M_{0},
$$

3. $\sqrt{n_{0}}, \sqrt{p_{0}}$ satisfy homogeneous Neumann boundary conditions, i.e.

$$
\sqrt{n_{0}}=-V\left[\sqrt{n_{0}}\left(\log \left(n_{0}\right)+V_{0}+\alpha_{0}\right)\right], \sqrt{p_{0}}=-V\left[\sqrt{p_{0}}\left(\log \left(p_{0}\right)-V_{0}+\beta_{0}\right)\right] .
$$


4. $p_{0} \in H^{1}(\Omega)$ and $\sqrt{p_{0}} \in H^{1}(\Omega)$.

The proof is deferred to the appendix.

Remark 6. a) Theorem 2 settles question a) of the introduction.

b) For the subsequent investigations of $\xi \rightarrow 0$ the result $\sqrt{p_{0}} \in H^{1}(\Omega)$ will be most important.

\section{Approximation by Quantum Models}

In this section we are concerned with the limiting behavior $\xi \rightarrow 0$ of the quintuple $\left(n_{\xi}, p_{\xi}, V_{\xi}, \alpha_{\xi}, \beta_{\xi}\right)$. Physically speaking the investigation addresses to the question whether the mixed quantum/classical state can be approximated by quantum/quantum states.

The main result is

Theorem 3. Assume (A1)-(A4). Then

1. The value of the ground energy of $J_{\xi}$ converges as $\xi \rightarrow 0$ to the value of the ground energy of $J_{0}$, i.e.

$$
\lim _{\xi \rightarrow 0}\left(\min _{\Gamma} J_{\xi}\right)=\min _{\Gamma_{0}} J_{0}
$$

and therefore

$$
\lim _{\xi \rightarrow 0} J_{\xi}\left(n_{\xi}, p_{\xi}\right)=J_{0}\left(n_{0}, p_{0}\right)
$$

2. There are positive $m, M$ such that

$$
\forall \xi \in[0, \infty): \quad m \leq n_{\xi}, p_{\xi} \leq M .
$$

3. $V_{\xi} \rightarrow V_{0}=V\left[n_{0}-p_{0}-C\right]$ strongly in $H^{1}(\Omega)$ and strongly in $L^{\infty}(\Omega)$ as $\xi \rightarrow 0$.

4. $n_{\xi} \rightarrow n_{0}$ strongly in $H^{1}(\Omega)$ and strongly in $L^{\infty}(\Omega)$ as $\xi \rightarrow 0$.

5. $p_{\xi} \rightarrow p_{0}$ strongly in $H^{1}(\Omega)$ and strongly in $L^{p}(\Omega), p \in[1, \infty)$, as $\xi \rightarrow 0$. Furthermore, $p_{\xi} \rightarrow p_{0}$ weak $^{*}$ in $L^{\infty}(\Omega)$ as $\xi \rightarrow 0$.

6. $\lim _{\xi \rightarrow 0} \alpha_{\xi}=\alpha_{0}$ and $\lim _{\xi \rightarrow 0} \beta_{\xi}=\beta_{0}$.

Remark 7. a) Theorem 3 settles questions b) and c) of the introduction.

b) The estimates of theorem 3 2. are independent of $\xi$. Thus the corresponding result of theorem 1 of [8] is improved. 
Proof. We observe $\Gamma \subseteq \Gamma_{0}$.

1. We have for all $\xi \in(0, \infty)$ and for all $(n, p) \in \Gamma$,

$$
J_{0}(n, p) \leq J_{\xi}(n, p), \quad \text { thus } \min _{\Gamma_{0}} J_{0} \leq \inf _{\Gamma} J_{0} \leq \min _{\Gamma} J_{\xi}
$$

and therefore

$$
\min _{\Gamma_{0}} J_{0} \leq \liminf _{\xi \rightarrow 0}\left(\min _{\Gamma} J_{\xi}\right)
$$

On the other hand we have $\left(n_{0}, p_{0}\right) \in \Gamma$ due to theorem 24 . Hence for all $\xi \in(0, \infty)$,

$$
\min _{\Gamma} J_{\xi} \leq J_{\xi}\left(n_{0}, p_{0}\right)
$$

Since $\lim _{\xi \rightarrow 0} J_{\xi}\left(n_{0}, p_{0}\right)=J_{0}\left(n_{0}, p_{0}\right)$, we have

$$
\limsup _{\xi \rightarrow 0}\left(\min _{\Gamma} J_{\xi}\right) \leq J_{0}\left(n_{0}, p_{0}\right)=\min _{\Gamma_{0}} J_{0}
$$

and therefore, due to (15) and (17), $\lim _{\xi \rightarrow 0}\left(\min _{\Gamma} J_{\xi}\right)=\min _{\Gamma_{0}} J_{0}$.

2. We shall derive some a priori estimates. Positive constants which are independent of $\xi$ are denoted by $K_{1}, K_{2}, K_{3}, \ldots$ For the sake of brevity we put for $t \geq 0, H(t):=t(\log (t)-1)$ (with $H(0)=0$ ). Then $H(t) \geq-1$ for all $t \geq 0$. We put $N_{0}:=\frac{N}{\operatorname{meas}(\Omega)}$ and $P_{0}:=\frac{P}{\operatorname{meas}(\Omega)}$. Then $\left(N_{0}, P_{0}\right) \in \Gamma \subseteq \Gamma_{0}$ and therefore, if $\xi \in(0, \infty)$ or $\xi=0$,

$$
\begin{aligned}
0 \leq J_{\xi}\left(n_{\xi}, p_{\xi}\right)+2 \operatorname{meas}(\Omega) & =\int_{\Omega}\left|\nabla \sqrt{n_{\xi}}\right|^{2} d x+\xi \int_{\Omega}\left|\nabla \sqrt{p_{\xi}}\right|^{2} d x \\
+\int_{\Omega}\left(H\left(n_{\xi}\right)+1\right) & d x+\int_{\Omega}\left(H\left(p_{\xi}\right)+1\right) d x+E_{\infty}\left(n_{\xi}-p_{\xi}-C\right) \\
& \leq J_{\xi}\left(N_{0}, P_{0}\right)+2 \operatorname{meas}(\Omega) \\
=: & K_{1}=\operatorname{meas}(\Omega)\left(H\left(N_{0}\right)+H\left(P_{0}\right)+2\right)+E_{\infty}\left(N_{0}-P_{0}-C\right) .
\end{aligned}
$$

By non-negativity of the terms of the left-hand side we deduce

$$
\forall \xi \in[0, \infty): \quad\left\|\sqrt{n_{\xi}}\right\|_{H^{1}(\Omega)} \leq K_{2}, \quad\left\|V_{\xi}\right\|_{H^{1}(\Omega)} \leq K_{2}
$$

where $V_{\xi}=V\left[n_{\xi}-p_{\xi}-C\right]$. Furthermore, we have due to $\left(n_{0}, p_{0}\right) \in \Gamma$ for all $\xi \in(0, \infty)$

$$
\begin{aligned}
J_{0}\left(n_{\xi}, p_{\xi}\right)+\xi \int_{\Omega}\left|\nabla \sqrt{p_{\xi}}\right|^{2} d x & =J_{\xi}\left(n_{\xi}, p_{\xi}\right) \\
& \leq J_{\xi}\left(n_{0}, p_{0}\right)=J_{0}\left(n_{0}, p_{0}\right)+\xi \int_{\Omega}\left|\nabla \sqrt{p_{0}}\right|^{2} d x
\end{aligned}
$$


while due to $\Gamma \subseteq \Gamma_{0}$,

$$
J_{0}\left(n_{0}, p_{0}\right) \leq J_{0}\left(n_{\xi}, p_{\xi}\right)
$$

We deduce from (20) and from (21) the estimate

$$
\forall \xi \in[0, \infty): \quad\left\|\sqrt{p_{\xi}}\right\|_{H^{1}(\Omega)} \leq K_{3}:=\left\|\sqrt{p_{0}}\right\|_{H^{1}(\Omega)} .
$$

From (19) and (22) we deduce via (A1)

$$
\forall \xi \in[0, \infty): \quad\left\|n_{\xi}\right\|_{L^{2}(\Omega)} \leq K_{4}, \quad\left\|p_{\xi}\right\|_{L^{2}(\Omega)} \leq K_{4} .
$$

Since $V_{\xi}=V\left[n_{\xi}-p_{\xi}-C\right]$ we deduce from (A4) via (23)

$$
\forall \xi \in[0, \infty): \quad\left\|V_{\xi}\right\|_{L^{\infty}(\Omega)} \leq K_{5} .
$$

Since $\sqrt{n_{\xi}} \in H^{1}(\Omega) \cap L^{\infty}(\Omega), \xi \in(0, \infty)$ or $\xi=0$, we can use $\sqrt{n_{\xi}}$ as test function in (2) to obtain

$$
\alpha_{\xi} N=-\int_{\Omega}\left|\nabla \sqrt{n_{\xi}}\right|^{2} d x-\int_{\Omega} n_{\xi} \log \left(n_{\xi}\right) d x-\int_{\Omega} V_{\xi} n_{\xi} d x
$$

hence by using $|t \log (t)| \leq 1+t+t^{2}$ for all $t \geq 0$,

$$
\left|\alpha_{\xi}\right| N \leq\left\|\sqrt{n_{\xi}}\right\|_{H^{1}(\Omega)}^{2}+1+N+\left\|n_{\xi}\right\|_{L^{2}(\Omega)}^{2}+\left\|V_{\xi}\right\|_{L^{2}(\Omega)}\left\|n_{\xi}\right\|_{L^{2}(\Omega)},
$$

and therefore via (19), (23),

$$
\forall \xi \in[0, \infty): \quad\left|\alpha_{\xi}\right| \leq K_{6} .
$$

Now we use for $\xi \in(0, \infty)$ (the case $\xi=0$ is excluded for the moment) the function $\sqrt{p_{\xi}} \in H^{1}(\Omega) \cap L^{\infty}(\Omega)$ as test function in (3) to obtain

$$
\beta_{\xi} P=-\xi \int_{\Omega}\left|\nabla \sqrt{p_{\xi}}\right|^{2} d x-\int_{\Omega} p_{\xi} \log \left(p_{\xi}\right) d x-\int_{\Omega} V_{\xi} p_{\xi} d x
$$

and we obtain by summing up (25) and (27),

$$
\begin{aligned}
\alpha_{\xi} N+ & \beta_{\xi} P \\
& =-J_{\xi}\left(n_{\xi}, p_{\xi}\right)+\int_{\Omega}\left(n_{\xi}+p_{\xi}\right) d x+\frac{1}{2}\left\|\mid \nabla V_{\xi}\right\|_{L^{2}(\Omega)}^{2}-\int_{\Omega} V_{\xi}\left(n_{\xi}+p_{\xi}\right) d x
\end{aligned}
$$

hence

$$
\begin{aligned}
\mid \alpha_{\xi} N & +\beta_{\xi} P \mid \\
& \leq\left|J_{\xi}\left(n_{\xi}, p_{\xi}\right)\right|+N+P+\left\|V_{\xi}\right\|_{H^{1}(\Omega)}^{2}+\left\|V_{\xi}\right\|_{L^{2}(\Omega)}\left(\left\|n_{\xi}\right\|_{L^{2}(\Omega)}+\left\|p_{\xi}\right\|_{L^{2}(\Omega)}\right)
\end{aligned}
$$


such that we obtain via (19), (23), (26) the estimate

$$
\forall \xi \in[0, \infty): \quad\left|\beta_{\xi}\right| \leq K_{7} .
$$

Now we can apply standard methods to deduce via (2), (3) for all $\xi \in(0, \infty)$,

$$
\begin{aligned}
& -\left\|V_{\xi}\right\|_{L^{\infty}(\Omega)}-\left|\alpha_{\xi}\right| \leq \log \left(n_{\xi}\right) \leq\left\|V_{\xi}\right\|_{L^{\infty}(\Omega)}+\left|\alpha_{\xi}\right|, \\
& -\left\|V_{\xi}\right\|_{L^{\infty}(\Omega)}-\left|\beta_{\xi}\right| \leq \log \left(p_{\xi}\right) \leq\left\|V_{\xi}\right\|_{L^{\infty}(\Omega)}+\left|\beta_{\xi}\right|,
\end{aligned}
$$

and therefore via (24), (26), (28),

$$
\forall \xi \in[0, \infty): \quad 0<K_{8} \leq n_{\xi}, p_{\xi} \leq K_{9}
$$

where the estimate for $\xi=0$ follows from theorem 2 .

It remains to prove the convergence statements. Let $\left(\xi_{k}\right)_{k \in \mathbb{N}}$ be a sequence in $(0, \infty)$ with $\lim _{k \rightarrow \infty} \xi_{k}=0$. For the sake of brevity we set

$$
n_{k}:=n_{\xi_{k}}, p_{k}:=p_{\xi_{k}}, V_{k}:=V_{\xi_{k}}, \alpha_{k}:=\alpha_{\xi_{k}}, \beta_{k}:=\beta_{\xi_{k}} .
$$

Due to (A1), (19), (22), (24), (26), (28), (29) we have - possibly after extracting a subsequence but without changing notations - $n_{*}, p_{*} \in H^{1}(\Omega) \cap L^{\infty}(\Omega)$ with $K_{8} \leq n_{*}, p_{*} \leq K_{9}, \alpha_{*}, \beta_{*} \in \mathbb{R}$ and $V_{*} \in H^{1}(\Omega) \cap L^{\infty}(\Omega)$ such that

$$
\begin{gathered}
V_{k} \rightarrow V_{*}, \sqrt{n_{k}} \rightarrow \sqrt{n_{*}}, \sqrt{p_{k}} \rightarrow \sqrt{p_{*}} \quad \text { weakly in } H^{1}(\Omega) \text { as } k \rightarrow \infty, \\
V_{k} \rightarrow V_{*}, n_{k} \rightarrow n_{*}, p_{k} \rightarrow p_{*} \quad \text { strongly in } L^{2}(\Omega) \text { as } k \rightarrow \infty, \\
V_{k} \rightarrow V_{*}, n_{k} \rightarrow n_{*}, p_{k} \rightarrow p_{*} \quad \text { almost everywhere as } k \rightarrow \infty, \\
V_{k} \rightarrow V_{*}, n_{k} \rightarrow n_{*}, p_{k} \rightarrow p_{*} \quad \text { weak }^{*} \text { in } L^{\infty}(\Omega) \text { as } k \rightarrow \infty, \\
\alpha_{k} \rightarrow \alpha_{*}, \beta_{k} \rightarrow \beta_{*} \quad \text { as } k \rightarrow \infty .
\end{gathered}
$$

We readily deduce from (32) and from (29):

$$
\forall p \in[1, \infty): \quad V_{k} \rightarrow V_{*}, n_{k} \rightarrow n_{*}, p_{k} \rightarrow p_{*} \quad \text { strongly in } L^{p}(\Omega) \text { as } k \rightarrow \infty
$$

and therefore due to (A4),

$$
V_{k} \rightarrow V_{*} \quad \text { strongly in } L^{\infty}(\Omega) \text { as } k \rightarrow \infty \text {. }
$$


Passing to the limit $k \rightarrow \infty$ in the weak formulation of the Poisson equation (3) we obtain

$$
V_{*}=V\left[n_{*}-p_{*}-C\right]
$$

as well as

$$
V_{k} \rightarrow V_{*} \quad \text { strongly in } H^{1}(\Omega) \text { as } k \rightarrow \infty,
$$

while by weak lower semi continuity,

$$
J_{0}\left(n_{*}, p_{*}\right) \leq \liminf _{k \rightarrow \infty} J_{\xi_{k}}\left(n_{k}, p_{k}\right)=\min _{\Gamma_{0}} J_{0}
$$

hence by $\left(n_{*}, p_{*}\right) \in \Gamma_{0}$ (which follows from (30) and (35))

$$
J_{0}\left(n_{*}, p_{*}\right)=\min _{\Gamma_{0}} J_{0}
$$

and therefore $\left(n_{*}, p_{*}\right)=\left(n_{0}, p_{0}\right)$ due to uniqueness of the minimizer in $\Gamma_{0}$. As a consequence, $V_{*}=V_{0}=V\left[n_{0}-p_{0}-C\right]$ and via (38),

$$
\int_{\Omega}\left|\nabla \sqrt{n_{k}}\right|^{2} d x \rightarrow \int_{\Omega}\left|\nabla \sqrt{n_{0}}\right|^{2} d x, \int_{\Omega}\left|\nabla \sqrt{p_{k}}\right|^{2} d x \rightarrow \int_{\Omega}\left|\nabla \sqrt{p_{0}}\right|^{2} d x \quad \text { as } k \rightarrow \infty
$$

hence

$$
\sqrt{n_{k}} \rightarrow \sqrt{n_{0}}, \sqrt{p_{k}} \rightarrow \sqrt{p_{0}} \text { strongly in } H^{1}(\Omega) \text { as } k \rightarrow \infty
$$

and therefore by (29),

$$
n_{k} \rightarrow n_{0}, p_{k} \rightarrow p_{0} \quad \text { strongly in } H^{1}(\Omega) \text { as } k \rightarrow \infty,
$$

as well. $\alpha_{*}=\alpha_{0}$ and $\beta_{*}=\beta_{0}$ follows from the previous estimates, from (25) and from (27). Finally, we deduce

$$
\begin{array}{r}
\sqrt{n_{k}}\left(\log \left(n_{k}\right)+V_{k}+\alpha_{k}\right) \rightarrow \sqrt{n_{0}}\left(\log \left(n_{0}\right)+V_{0}+\alpha_{0}\right) \\
\text { strongly in } L^{2}(\Omega) \text { as } k \rightarrow \infty,
\end{array}
$$

hence, due to $\sqrt{n_{\xi}}=V\left[\sqrt{n_{k}}\left(\log \left(n_{k}\right)+V_{k}+\alpha_{k}\right)\right]$, and due to $\sqrt{n_{0}}=V\left[\sqrt{n_{0}}\left(\log \left(n_{0}\right)+V_{0}+\alpha_{0}\right)\right]$, we deduce from (A4), $n_{k} \rightarrow n_{0}$ strongly in $L^{\infty}(\Omega)$ as $k \rightarrow \infty$.

\section{Appendix: Minimization of $J_{0}$ in $\Gamma_{0}$}

Proof. (Theorem 2) The verification that $J_{0}$ has a minimizer in $\Gamma_{0}$ may be settled on more or less standard arguments involving coercitivity and weak lower semicontinuity. However the derivation of the associated Euler Lagrange is due to the lack of differentiability of the function $t \mapsto t \log (t)$ at $t=0$ not straight-forward. 
We proceed by an approximation argument.

For $\delta \in(0,1)$ and $t \in \mathbb{R}$ let

$$
\log _{\delta}(t)=\left\{\begin{array}{rl}
\log (t) & \text { if } t>\delta \\
2 t-2 \delta+\log (\delta) & \text { if } t \leq \delta
\end{array},\right.
$$

and

$$
H_{\delta}(t)=\left\{\begin{array}{rl}
t(\log (t)-1) & \text { if } t>\delta \\
t^{2}+(\log (\delta)-2 \delta) t+\delta^{2}-\delta \log (\delta) & \text { if } t \leq \delta
\end{array},\right.
$$

such that $H_{\delta}$ is continuously differentiable on $\mathbb{R}$ with derivative $\log _{\delta}$. We note

$$
-1 \leq H_{\delta}(t) \leq H(t):=t(\log (t)-1), \quad \forall t \geq 0, \forall \delta \in(0,1) .
$$

We introduce [6]

$$
\begin{aligned}
\Gamma^{*}:=\left\{(\rho, p) \in H^{1}(\Omega) \times \mathcal{C}_{e l}:\right. & \\
& \left.\left(\int_{\Omega} \rho \rho^{+} d x=N\right) \wedge(p \geq 0) \wedge\left(\int_{\Omega} p d x=P\right)\right\},
\end{aligned}
$$

where

$$
\rho^{+}=\max \{\rho, 0\}, \quad \rho^{-}=\max \{-\rho, 0\} .
$$

For $\delta \in(0,1)$ we define the functional

$$
\begin{aligned}
& E_{\delta}: \Gamma^{*} \rightarrow \mathbb{R} \cup\{\infty\}, \\
& \qquad \begin{aligned}
E_{\delta}(\rho, p)=\int_{\Omega}|\nabla \rho|^{2} d x+\int_{\Omega} \rho \rho^{+}\left(\log \left(\rho \rho^{+}\right)-1\right) d x & +\int_{\Omega} H_{\delta}(p) d x \\
& +E_{\infty}\left(\rho \rho^{+}-p-C\right) .
\end{aligned}
\end{aligned}
$$

Furthermore, we introduce

$$
\begin{aligned}
& E_{0}: \Gamma^{*} \rightarrow \mathbb{R} \cup\{\infty\}, \\
& \qquad \begin{aligned}
E_{0}(\rho, p)=\int_{\Omega}|\nabla \rho|^{2} d x+\int_{\Omega} \rho \rho^{+}\left(\log \left(\rho \rho^{+}\right)-1\right) d x+ & \int_{\Omega} p(\log (p)-1) d x \\
& +E_{\infty}\left(\rho \rho^{+}-p-C\right) .
\end{aligned}
\end{aligned}
$$

Our strategy is to prove existence and uniqueness of a minimizer $\left(\rho_{\delta}, p_{\delta}\right)$ of $E_{\delta}$ in $\Gamma^{*}$, to justify the validity of the corresponding Euler Lagrange equations, to derive $\delta$-independent estimates and to pass to the limit $\delta \rightarrow 0$ then. 
Step 1: Uniqueness of a minimizer. Let $\delta \in(0,1)$ or $\delta=0$ be fixed for the moment. Since $E_{\delta}$ is not convex, uniqueness of a minimizer of $E_{\delta}$ in $\Gamma^{*}$ is a priori not clear. Assume $\left(\rho_{\delta}, p_{\delta}\right)$ and $\left(\rho_{\circ}, p_{\circ}\right)$ are minimizers of $E_{\delta}$ in $\Gamma^{*}$, i.e.

$$
E_{\delta}\left(\rho_{\delta}, p_{\delta}\right)=E_{\delta}\left(\rho_{\circ}, p_{\circ}\right)=\inf _{\Gamma^{*}} E_{\delta},
$$

where we note $\inf _{\Gamma^{*}} E_{\delta} \geq-2$.

We set

$$
\rho_{\theta}:=\sqrt{(1-\theta) \rho_{\delta} \rho_{\delta}^{+}+\theta \rho_{\circ} \rho_{\circ}^{+}}, \quad p_{\theta}:=(1-\theta) p_{\delta}+\theta p_{\circ}, \quad \theta \in(0,1) .
$$

Then $\left(\rho_{\theta}, p_{\theta}\right) \in \Gamma^{*}$ for all $\theta \in(0,1)$. Since $\rho_{\theta} \rho_{\theta}^{+}=(1-\theta) \rho_{\delta} \rho_{\delta}^{+}+\theta \rho_{\circ} \rho_{\circ}^{+}$by strict convexity,

$$
\begin{gathered}
\int_{\Omega} \rho_{\theta} \rho_{\theta}^{+}\left(\log \left(\rho_{\theta} \rho_{\theta}^{+}\right)-1\right) d x+\int_{\Omega} H_{\delta}\left(p_{\theta}\right) d x+E_{\infty}\left(\rho_{\theta} \rho_{\theta}^{+}-p_{\theta}-C\right) \\
<(1-\theta)\left(\int_{\Omega} \rho_{\delta} \rho_{\delta}^{+}\left(\log \left(\rho_{\delta} \rho_{\delta}^{+}\right)-1\right) d x+\int_{\Omega} H_{\delta}\left(p_{\delta}\right) d x+E_{\infty}\left(\rho_{\delta} \rho_{\delta}^{+}-p_{\delta}-C\right)\right)+ \\
\quad \theta\left(\int_{\Omega} \rho_{\circ} \rho_{\circ}^{+}\left(\log \left(\rho_{\circ} \rho_{\circ}^{+}\right)-1\right) d x+\int_{\Omega} H_{\delta}\left(p_{\circ}\right) d x+E_{\infty}\left(\rho_{\circ} \rho_{\circ}^{+}-p_{\circ}-C\right)\right),
\end{gathered}
$$

for all $\theta \in(0,1)$ unless $\left(\rho_{\delta}, p_{\delta}\right)=\left(\rho_{\circ}, p_{\circ}\right)$. Furthermore, we have for all $\theta \in(0,1)$ the inequality

$$
\begin{aligned}
(1-\theta) \int_{\Omega}\left|\nabla \rho_{\delta}\right|^{2} d x & +\theta \int_{\Omega}\left|\nabla \rho_{\circ}\right|^{2} d x-\int_{\Omega}\left|\nabla \rho_{\theta}\right|^{2} d x \\
& =\theta(1-\theta) \int_{\left\{\rho_{\circ}^{+}>0\right\} \cup\left\{\rho_{\delta}^{+}>0\right\}} \frac{\left(\rho_{\circ}^{+} \nabla \rho_{\delta}^{+}-\rho_{\delta}^{+} \nabla \rho_{\circ}^{+}\right)^{2}}{(1-\theta) \rho_{\delta} \rho_{\delta}^{+}+\theta \rho_{\circ} \rho_{\circ}^{+}} d x \geq 0,
\end{aligned}
$$

hence

$$
E_{\delta}\left(\rho_{\theta}, p_{\theta}\right)<(1-\theta) E_{\delta}\left(\rho_{\delta}, p_{\delta}\right)+\theta E_{\delta}\left(\rho_{\circ}, p_{\circ}\right), \quad \theta \in(0,1)
$$

unless $\left(\rho_{\delta}, p_{\delta}\right)=\left(\rho_{\circ}, p_{\circ}\right)$.

Step 2: Existence of a minimizer. Let $\delta \in(0,1)$ or $\delta=0$ be fixed for the moment. We consider a minimizing sequence $\left(\rho_{k}, p_{k}\right)_{k \in \mathbb{N}}$. Since $E\left(\rho_{k}^{+}, p_{k}\right) \leq E\left(\rho_{k}, p_{k}\right)$ and since $\left(\rho_{k}^{+}, p_{k}\right) \in \Gamma^{*}$ whenever $\left(\rho_{k}, p_{k}\right) \in \Gamma^{*}$, we can assume $\rho_{k}=\rho_{k}^{+} \geq 0$ for all $k \in \mathbb{N}$. Hence

We observe $\left(\sqrt{N_{0}}, P_{0}\right) \in \Gamma^{*}$, where $N_{0}:=N / \operatorname{meas}(\Omega)$ and $P_{0}:=P / \operatorname{meas}(\Omega)$.

$$
\begin{aligned}
& \inf _{\Gamma^{*}} E_{\delta} \leq E_{\delta}\left(\sqrt{N_{0}}, P_{0}\right)<E_{\delta}\left(\sqrt{N_{0}}, P_{0}\right)+1=K_{1} \\
& :=1+\left(N_{0}\left(\log \left(N_{0}\right)-1\right)+P_{0}\left(\log \left(P_{0}\right)-1\right)\right) \operatorname{meas}(\Omega)+E_{\infty}\left(N_{0}-P_{0}-C\right),
\end{aligned}
$$


where we have made use of (41). Due to assumption (A4) we have $K_{1} \in \mathbb{R}$. We note: $K_{1}$ does not depend on $\delta$.

We assume $E_{\delta}\left(\rho_{k}, p_{k}\right) \leq K_{1}$ for all $k \in \mathbb{N}$. Then we have due to (42) and due to (41) the a priori estimate

$$
\begin{array}{r}
\int_{\Omega}\left|\nabla \rho_{k}\right|^{2} d x+\int_{\Omega}\left(\rho_{k}^{2}\left(\log \left(\rho_{k}^{2}\right)-1\right)+1\right) d x+\int_{\Omega}\left(H_{\delta}\left(p_{k}\right)+1\right) d x+E_{\infty}\left(\rho_{k}^{2}-p_{k}-C\right) \\
\leq K_{2}:=K_{1}+2 \operatorname{meas}(\Omega)
\end{array}
$$

where $K_{2}$ is independent of $\delta$.

Since all terms on the left hand side or this inequality ro non negatve we have by passing if necessary to a subsequence but without changing notations

$$
\begin{gathered}
\rho_{k}=\rho_{k}^{+} \rightarrow \rho_{\delta} \quad \text { weakly in } H^{1}(\Omega) \text { as } k \rightarrow \infty, \\
p_{k} \rightarrow p_{\delta} \quad \text { weakly in } L^{1}(\Omega) \text { as } k \rightarrow \infty,
\end{gathered}
$$

and therefore by assumption (A1),

$$
\begin{aligned}
& \rho_{k} \rightarrow \rho_{\delta} \quad \text { strongly in } L^{4}(\Omega) \text { as } k \rightarrow \infty, \\
& \rho_{k}^{2} \rightarrow \rho_{\delta}^{2} \quad \text { strongly in } L^{2}(\Omega) \text { as } k \rightarrow \infty .
\end{aligned}
$$

As a consequence, $\rho_{\delta} \geq 0$ and $\left(\rho_{\delta}, p_{\delta}\right) \in \Gamma^{*}$. We certainly have

$$
\int_{\Omega}\left|\nabla \rho_{\delta}\right|^{2} d x \leq \liminf _{k \rightarrow \infty} \int_{\Omega}\left|\nabla \rho_{k}\right|^{2} d x
$$

and due to $\rho_{k} \rho_{k}^{+}=\rho_{k}^{2}, k \in \mathbb{N}$, due to $\rho_{\delta} \rho_{\delta}^{+}=\rho_{\delta}^{2}$, by convexity

$$
\begin{aligned}
\int_{\Omega} \rho_{\delta}^{2}\left(\log \left(\rho_{\delta}^{2}\right)-1\right) d x & +\int_{\Omega} H_{\delta}\left(p_{\delta}\right) d x \\
& \leq \liminf _{k \rightarrow \infty}\left(\int_{\Omega} \rho_{k}^{2}\left(\log \left(\rho_{k}^{2}\right)-1\right) d x+\int_{\Omega} H_{\delta}\left(p_{k}\right) d x\right) .
\end{aligned}
$$

Now let us consider $E_{\infty}\left(\rho_{k}^{2}-p_{k}-C\right)$ as $k \rightarrow \infty$. Since a priori only $\rho_{k}^{2}-p_{k}-C \rightarrow$ $\rho_{\delta}^{2}-p_{\delta}-C$ weakly in $L^{1}(\Omega)$ as $k \rightarrow \infty$, the statement $\rho_{\delta}^{2}-p_{\delta}-C \in \mathcal{C}_{e l}$ (compare remark $2 \mathrm{c}$ ) it is not immediate. We set $V_{k}:=V\left[\rho_{k}^{2}-p_{k}-C\right]$ for $k \in \mathbb{N}$. Since the sequence $\left(\left\|\nabla V_{k}\right\|_{L^{2}(\Omega): \mathbb{R}^{d}}\right)_{k \in \mathbb{N}}$ is bounded (see (43)) we have (possibly after passing to a subsequence, but without changing notations) due to $\int_{\Omega} V_{k} d x=0$ for all $k \in \mathbb{N}$,

$$
V_{k} \rightarrow V_{\delta}, \quad \text { weakly in } H^{1}(\Omega) \text { as } k \rightarrow \infty,
$$


which implies

$$
\int_{\Omega}\left|\nabla V_{\delta}\right|^{2} d x \leq \liminf _{k \rightarrow \infty} \int_{\Omega}\left|\nabla V_{k}\right|^{2} d x
$$

while on the other hand for all $\phi \in \mathcal{C}_{0} \cap L^{\infty}(\Omega)$,

$$
\begin{aligned}
\int_{\Omega} \nabla V_{\delta} \cdot \nabla \phi d x=\lim _{k \rightarrow \infty} & \int_{\Omega} \nabla V_{k} \cdot \nabla \phi d x \\
& =\lim _{k \rightarrow \infty} \int_{\Omega}\left(\rho_{k}^{2}-p_{k}-C\right) \phi d x=\int_{\Omega}\left(\rho_{\delta}^{2}-p_{\delta}-C\right) \phi d x,
\end{aligned}
$$

such that we deduce $\rho_{\delta}^{2}-p_{\delta}-C \in \mathcal{C}_{e l}, V_{\delta}=V\left[\rho_{\delta}^{2}-p_{\delta}-C\right]$, via (46), and via

$$
E_{\infty}\left(\rho_{\delta}^{2}-p_{\delta}-C\right) \leq \liminf _{k \rightarrow \infty} E_{\infty}\left(\rho_{k}^{2}-p_{k}-C\right)
$$

the estimate

$$
E_{\delta}\left(\rho_{\delta}, p_{\delta}\right) \leq \liminf _{k \rightarrow \infty} E_{\delta}\left(\rho_{k}, p_{k}\right),
$$

i.e. $\left(\rho_{\delta}, p_{\delta}\right)$ is a minimizer of $E_{\delta}$ in $\Gamma^{*}$.

Step 3: Derivation of the Euler Lagrange equations. Here we make use of the differentiability of $H_{\delta}$. Hence the case $\delta=0$ is excluded.

Let $\delta \in(0,1)$ be fixed for the moment. We shall derive the Euler Lagrange equations for $\left(\rho_{\delta}, p_{\delta}\right)$.

Let $\phi \in H^{1}(\Omega) \cap L^{\infty}(\Omega)$. For $t \in(0,1)$ let

$$
N(t):=\sqrt{\int_{\Omega}\left(\rho_{\delta}+t \phi\right)\left(\rho_{\delta}+t \phi\right)^{+} d x} .
$$

We certainly have $N(0+)=N$. In particular, there is $t_{0} \in(0,1)$ such that $N(t) \geq N / 2$ for all $t \in\left(0, t_{0}\right]$. We set

$$
\phi_{t}:=\frac{N}{N(t)}\left(\rho_{\delta}+t \phi\right), \quad t \in\left(0, t_{0}\right]
$$

Obviously, $\phi_{t} \in H^{1}(\Omega)$ with $\int_{\Omega} \phi_{t} \phi_{t}^{+} d x=N, t \in\left(0, t_{0}\right]$. As a consequence, we have $\left(\phi_{t}, p_{\delta}\right) \in \Gamma^{*}$ for all $t \in\left(0, t_{0}\right]$. Furthermore it is easy to see

$$
\infty>E_{\delta}\left(\phi_{t}, p_{\delta}\right)-E_{\delta}\left(\rho_{\delta}, p_{\delta}\right) \geq 0, \quad t \in\left(0, t_{0}\right]
$$

thus

$$
\liminf _{t \rightarrow 0+} \frac{E_{\delta}\left(\phi_{t}, p_{\delta}\right)-E_{\delta}\left(\rho_{\delta}, p_{\delta}\right)}{t} \geq 0
$$


Since

$$
N^{2}(t)=N^{2}+2 t \int_{\Omega} \rho_{\delta} \phi d x+t^{2} \int_{\Omega} A\left(\rho_{\delta}(x), \phi(x)\right) \phi^{2}(x) d x, \quad t \in\left(0, t_{0}\right]
$$

for a measurable function $A: \mathbb{R}^{2} \rightarrow \mathbb{R}$ with $\left|A\left(\sigma_{1}, \sigma_{2}\right)\right| \leq 2$ for all $\left(\sigma_{1}, \sigma_{2}\right) \in \mathbb{R}^{2}$, we obtain

$$
\begin{aligned}
& \lim _{t \rightarrow 0+} \frac{\int_{\Omega}\left(\left|\nabla \phi_{t}\right|^{2}-\left|\nabla \rho_{\delta}\right|^{2}\right) d x}{t} \\
&=-\frac{1}{N^{2}} \int_{\Omega} \rho_{\delta} \phi d x \int_{\Omega}\left|\nabla \rho_{\delta}\right|^{2} d x+2 \int_{\Omega} \nabla \rho_{\delta} \cdot \nabla \phi d x .
\end{aligned}
$$

We deduce from the estimate

$$
\forall \sigma \geq 0: 4|\sigma \log (\sigma)| \leq 4\left(1+\sigma+\sigma^{2}\right)
$$

due to $\rho_{\delta}, \phi \in L^{6}(\Omega)$, see (A1), for all $t \in\left(0, t_{0}\right]$ the estimate

$$
\left|\frac{H\left(\phi_{t}\right)-H\left(\rho_{\delta}^{2}\right)}{t}\right| \leq\left(1+\frac{N}{N(t)}\right)^{3}\left(a_{0}+a_{1}\left(\rho_{\delta}^{6}+|\phi|^{6}\right)\right)
$$

where $H(\sigma)=\sigma^{2}\left(\log \left(\sigma^{2}\right)-1\right), \sigma \geq 0$, and $a_{0}, a_{1}$ are some positive constants which are independent of $\rho_{\delta}, \Phi$.

Now it is easy to deduce

$$
\begin{aligned}
\lim _{t \rightarrow 0+} \int_{\Omega} \frac{H\left(\phi_{t}\right)-H\left(\rho_{\delta}\right)}{t} d x \\
=\int_{\Omega}\left(2 \rho_{\delta} \log \left(\rho_{\delta}^{2}\right)\right) \phi d x-\frac{1}{N^{2}} \int_{\Omega} \rho_{\delta} \phi d x \int_{\Omega} 2 \rho_{\delta}^{3} \log \left(\rho_{\delta}^{2}\right) d x
\end{aligned}
$$

via Lebesgue's dominated convergence theorem.

Furthermore, we obtain with the aid of remark 2 c) that

$$
\begin{aligned}
& \lim _{t \rightarrow 0+} \frac{E_{\infty}\left(\phi_{t}-p_{\delta}-C\right)-E_{\infty}\left(\rho_{\delta}^{2}-p_{\delta}\right)}{t} \\
&=2 \int_{\Omega} V_{\delta} \rho_{\delta} \phi d x-\frac{1}{N^{2}} \int_{\Omega} \rho_{\delta} \phi d x \int_{\Omega} V_{\delta} \rho_{\delta}^{2} d x .
\end{aligned}
$$

Equations (48), (49), (50) hold for all $\phi \in H^{1}(\Omega) \cap L^{\infty}(\Omega)$. Hence we have actually equality in (47) and therefore

$$
\int_{\Omega} \nabla \rho_{\delta} \cdot \nabla \phi d x+\int_{\Omega} \rho_{\delta}\left(\log \left(\rho_{\delta}^{2}\right)+V_{\delta}+\alpha_{\delta}\right) \phi d x=0,
$$


where $\alpha_{\delta} \in \mathbb{R}$ is given by

$$
\alpha_{\delta}=-\frac{1}{2 N^{2}}\left(\int_{\Omega}\left|\nabla \rho_{\delta}\right|^{2} d x+2 \int_{\Omega} \rho_{\delta}^{3} \log \left(\rho_{\delta}^{2}\right) d x+\int_{\Omega} V_{\delta} \rho_{\delta}^{2} d x\right) .
$$

By a similiar, but standard procedure [5] (here the differentiability of $H_{\delta}$ enters) we deduce the variational inequalities

$$
\begin{array}{lll}
\log _{\delta}\left(p_{\delta}\right)-V_{\delta}+\beta_{\delta}=0 & \text { on } & \left\{p_{\delta}>0\right\} \\
\log _{\delta}(0)-V_{\delta}+\beta_{\delta} \geq 0 & \text { on } & \left\{p_{\delta}=0\right\}
\end{array}
$$

with $\beta_{\delta} \in \mathbb{R}$ follow.

Introducing the generalized inverse of $\log _{\delta}^{+}$,

$$
g_{\delta}: \mathbb{R} \rightarrow[0, \infty), \quad g_{\delta}(\sigma)=\left\{\begin{array}{ccc}
\log _{\delta}^{-1}(\sigma) & \text { if } & \sigma>-2 \delta+\log (\delta) \\
0 & \text { if } & \sigma \leq-2 \delta+\log (\delta)
\end{array}\right.
$$

we rewrite (53) as

$$
p_{\delta}=g_{\delta}\left(V_{\delta}-\beta_{\delta}\right)
$$

Step 4: Estimates independent of $\delta \in(0,1)$. Several constants which are independent of $\delta$ are denoted by $K_{3}, K_{4}, \ldots$.

We deduce from (43), (44), (45) and (46) the estimate

$$
\begin{array}{r}
\int_{\Omega}\left|\nabla \rho_{\delta}\right|^{2} d x+\int_{\Omega}\left(\rho_{\delta}^{2}\left(\log \left(\rho_{\delta}^{2}\right)-1\right)+1\right) d x+\int_{\Omega}\left(H_{\delta}\left(p_{\delta}\right)+1\right) d x+E_{\infty}\left(\rho_{\delta}^{2}-p_{\delta}-C\right) \\
\leq K_{2}, \quad(55)
\end{array}
$$

where $K_{2}$ is independent of $\delta$. In particular we deduce

$$
\left\|\rho_{\delta}\right\|_{H^{1}(\Omega)}^{2} \leq K_{3}:=N+K_{2}, \quad\left\|V_{\delta}\right\|_{H^{1}(\Omega)} \leq K_{4},
$$

from which we deduce due to (52), and due to (A1) for some (products of) imbedding constants $a_{0}, a_{1}, a_{2}, a_{3} \in(0, \infty)$ (which are intrinisically independent of $\delta$ ),

$$
\begin{gathered}
\left|\alpha_{\delta}\right| \leq \frac{1}{2 N^{2}}\left(\left\|\rho_{\delta}\right\|_{H^{1}(\Omega)}^{2}+\int_{\Omega}\left(1+\rho_{\delta}^{3}+\rho_{\delta}^{6}\right) d x+\left\|V_{\delta}\right\|_{L^{2}(\Omega)}\left\|\rho_{\delta}\right\|_{L^{4}(\Omega)}\right) \\
\leq \frac{1}{2 N^{2}}\left(\left\|\rho_{\delta}\right\|_{H^{1}(\Omega)}^{2}+a_{0}+a_{1}\left\|\rho_{\delta}\right\|_{H^{1}(\Omega)}^{3}+a_{2}\left\|\rho_{\delta}\right\|_{H^{1}(\Omega)}^{6}+a_{3}\left\|V_{\delta}\right\|_{H^{1}(\Omega)}\left\|\rho_{\delta}\right\|_{H^{1}(\Omega)}\right) \\
\leq K_{5}:=\frac{1}{2 N^{2}}\left(K_{3}+a_{0}+a_{1} K_{3}^{3 / 2}+a_{2} K_{3}^{3}+a_{3} \sqrt{K_{3}} K_{4}\right)
\end{gathered}
$$


In a similiar we proceed to prove

$$
\forall \delta \in(0,1): \quad \rho_{\delta}\left(\log \left(\rho_{\delta}^{2}\right)+V_{\delta}+\alpha_{\delta}\right) \in L^{2}(\Omega),
$$

with

$$
\left\|\rho_{\delta}\left(\log \left(\rho_{\delta}^{2}\right)+V_{\delta}+\alpha_{\delta}\right)\right\|_{L^{2}(\Omega)} \leq K_{6} .
$$

Furthermore, (51) holds for all $\phi \in H^{1}(\Omega) \cap L^{\infty}(\Omega)$. Hence

$$
\rho_{\delta}=V\left[\rho_{\delta}\left(\log \left(\rho_{\delta}^{2}\right)+V_{\delta}+\alpha_{\delta}\right)\right],
$$

and therefore due to (A4),

$$
\left\|\rho_{\delta}\right\|_{L^{\infty}(\Omega)} \leq K_{7}:=K_{\infty} K_{6} .
$$

Due to (54) we have

$$
-\Delta V_{\delta}=\rho_{\delta}^{2}-g_{\delta}\left(V_{\delta}-\beta_{\delta}\right)-C .
$$

Since $\lim _{\sigma \rightarrow \infty} g_{\delta}(\sigma)=\infty$, there is $m \in(0, \infty)$ with $g_{\delta}(m)=1+K_{7}^{2}+\|C\|_{L^{\infty}(\Omega)}$. The function $\left[V_{\delta}-\beta_{\delta}-m\right]^{+}$belongs to $H^{1}(\Omega)$. We take for $k \in \mathbb{N}$,

$$
\phi_{k}:=\min \left\{k,\left[V_{\delta}-\beta_{\delta}-m\right]^{+}\right\} \in H^{1}(\Omega) \cap L^{\infty}(\Omega) .
$$

Certainly, $\phi_{k} \rightarrow\left[V_{\delta}-\beta_{\delta}-m\right]^{+}$as $k \rightarrow \infty$, strongly in $H^{1}(\Omega)$ with $0 \leq \phi_{k} \leq \phi_{k+1}$ for all $k \in \mathbb{N}$. Hence for all $k \in \mathbb{N}$,

$$
\begin{aligned}
-\int_{\Omega} \nabla V_{\delta} \cdot \nabla \phi_{k} d x=-\int_{\Omega} & \left(\rho_{\delta}^{2}-g_{\delta}\left(V_{\delta}-\beta_{\delta}\right)-C\right) \phi_{k} d x \\
& =\int_{\left\{V_{\delta}-\beta_{\delta} \geq m\right\}}\left(-\rho_{\delta}^{2}+g_{\delta}\left(V_{\delta}-\beta_{\delta}\right)+C\right) \phi_{k} d x .
\end{aligned}
$$

By strong convergence in $H^{1}(\Omega)$ we have

$$
\begin{aligned}
\lim _{k \rightarrow \infty} \int_{\Omega} \nabla V_{\delta} \cdot \nabla \phi_{k} d x=\int_{\Omega} \nabla V_{\delta} \cdot \nabla\left[V_{\delta}-\beta_{\delta}-m\right]^{+} & d x \\
& =\int_{\Omega}\left|\nabla\left[V_{\delta}-\beta_{\delta}-m\right]^{+}\right|^{2} d x,
\end{aligned}
$$

hence by Beppo Levi's theorem (we note $\left(-\rho_{\delta}^{2}+g_{\delta}\left(V_{\delta}-\beta_{\delta}\right)+C\right) \phi_{k} \geq 0$ on $\left.\left\{V_{\delta}-\beta_{\delta} \geq m\right\}\right)$,

$$
\begin{aligned}
\lim _{k \rightarrow \infty} \int_{\left\{V_{\delta}-\beta_{\delta} \geq m\right\}}\left(-\rho_{\delta}^{2}\right. & \left.+g_{\delta}\left(V_{\delta}-\beta_{\delta}\right)+C\right) \phi_{k} d x \\
& =\int_{\left\{V_{\delta}-\beta_{\delta} \geq m\right\}}\left(-\rho_{\delta}^{2}+g_{\delta}\left(V_{\delta}-\beta_{\delta}\right)+C\right)\left[V_{\delta}-\beta_{\delta}-m\right]^{+} d x .
\end{aligned}
$$


Now we deduce from (60),

$$
0 \geq \int_{\left\{V_{\delta}-\beta_{\delta} \geq m\right\}}\left(-\rho_{\delta}^{2}+g_{\delta}\left(V_{\delta}-\beta_{\delta}\right)+C\right)\left[V_{\delta}-\beta_{\delta}-m\right]^{+} d x \geq 0
$$

and therefore $\left[V_{\delta}-\beta_{\delta}-m\right]^{+}=0$ almost everywhere on $\Omega$. Thus, $V_{\delta} \leq m+\beta_{\delta}$ almost everywhere on $\Omega$. As a consequence, since $g_{\delta}$ is strictly increasing,

$$
p_{\delta}=g_{\delta}\left(V_{\delta}-\beta_{\delta}\right) \leq g_{\delta}(m)=K_{8}:=1+K_{7}^{2}+\|C\|_{L^{\infty}(\Omega)} .
$$

On the other hand, $V_{\delta}=V\left[\rho_{\delta}^{2}-p_{\delta}-C\right]$, such that we deduce from (A4)

$$
\begin{aligned}
\left\|V_{\delta}\right\|_{L^{\infty}(\Omega)} \leq K_{\infty} \| \rho_{\delta}^{2}-p_{\delta} & -C \|_{L^{2}(\Omega)} \\
& \leq K_{9}:=K_{\infty} \sqrt{\operatorname{meas}(\Omega)}\left(K_{7}^{2}+K_{8}+\|C\|_{L^{\infty}(\Omega)}\right) .
\end{aligned}
$$

Since $\left\{p_{\delta}>0\right\}$ has nonzero measure we deduce from (53),

$$
-\beta_{\delta} \leq K_{10}:=\left\|V_{\delta}\right\|_{L^{\infty}(\Omega)}+\log _{\delta}\left(K_{8}\right)=\left\|V_{\delta}\right\|_{L^{\infty}(\Omega)}+\log \left(K_{8}\right),
$$

because due to (61) we have $K_{8} \geq 1$ and by construction we have $\log _{\delta}(\sigma)=\log (\sigma)$ for all $\sigma \geq 1$.

On the other hand it is easy to see that

$$
g_{\delta}(\sigma) \leq \exp (\sigma), \quad \sigma \in \mathbb{R} .
$$

Hence

$$
P=\int_{\Omega} g_{\delta}\left(V_{\delta}-\beta_{\delta}\right) d x \leq \int_{\Omega} \exp \left(V_{\delta}-\beta_{\delta}\right) d x \leq \int_{\Omega} \exp \left(K_{9}-\beta_{\delta}\right) d x
$$

from which we deduce

$$
\beta_{\delta} \leq K_{11}:=|\log (P)|+K_{9}+|\log (\operatorname{meas}(\Omega))| .
$$

We can summarize (63) and (64) as

$$
\left|\beta_{\delta}\right| \leq K_{12}:=K_{10}+K_{11}
$$

Step 5: Passing from $E_{\delta}$ to $E_{0}$. According to (53) there is $\delta^{*} \in(0,1)$ such that $\overline{p_{\delta}>0}$ almost everywhere on $\Omega$ for all $\delta \in\left(0, \delta^{*}\right)$, because otherwise $-2 \delta+\log (\delta) \geq$ $-K_{12}-K_{9}$ for all $\delta \in(0,1)$. We therefore have $\log _{\delta}\left(p_{\delta}\right)-V_{\delta}+\beta_{\delta}=0$ for all $\delta \in\left(0, \delta^{*}\right)$. Since $\log _{\delta}(\sigma) \leq \log (\delta)$ for all $\sigma \leq \delta$ and since $\left\|V_{\delta}\right\|_{L^{\infty}(\Omega)}+\left|\beta_{\delta}\right| \leq$ $K_{9}+K_{12}$, we deduce: there is $\delta_{1} \in\left(0, \delta^{*}\right)$ such that $p_{\delta} \geq \delta_{1}$ for all $\delta \in\left(0, \delta_{1}\right)$. Hence $\log _{\delta}\left(p_{\delta}\right)=\log \left(p_{\delta}\right)$ for all $\delta \in\left(0, \delta_{1}\right)$. As a consequence, we have

$$
p_{\delta}\left(\log \left(p_{\delta}\right)-1\right)=H_{0}\left(p_{\delta}\right)=H_{\delta}\left(p_{\delta}\right), \quad \delta \in\left(0, \delta_{1}\right) .
$$


Hence

$$
E_{\delta}\left(\rho_{\delta}, p_{\delta}\right)=E_{0}\left(\rho_{\delta}, p_{\delta}\right), \quad \delta \in\left(0, \delta_{1}\right)
$$

On the other hand, for all $(\rho, p) \in \Gamma^{*}$, we have due to (41)

$$
E_{\delta}(\rho, p) \leq E_{0}(\rho, p)
$$

Hence for all $\delta \in\left(0, \delta_{1}\right)$,

$$
\inf _{\Gamma^{*}} E_{0} \leq E_{0}\left(\rho_{\delta}, p_{\delta}\right)=E_{\delta}\left(\rho_{\delta}, p_{\delta}\right)=\inf _{\Gamma^{*}} E_{\delta} \leq \inf _{\Gamma^{*}} E_{0}
$$

and as a conclusion: There is $\delta_{\circ} \in(0,1)$ such that $\left(\rho_{\delta_{0}}, p_{\delta_{\circ}}\right)$ is the unique minimizer of $E_{0}$ in $\Gamma^{*}$. We set $\left(\rho_{2}, p_{2}\right):=\left(\rho_{\delta_{\circ}}, p_{\delta_{\circ}}\right)$.

Step 6: Finishing the proof. We have to prove that $\left(\rho_{2}^{2}, p_{2}\right)$ is the unique minimizer $\overline{\text { of } J_{0} \text { in }} \Gamma_{0}$. Since $(\sqrt{n}, p) \in \Gamma^{*}$ and since $E_{0}(\sqrt{n}, p)=J_{0}(n, p)$ for all $(n, p) \in \Gamma^{*}$ we have

$$
\inf _{\Gamma^{*}} E_{0} \leq \inf _{\Gamma_{0}} J_{0}
$$

On the other hand we have $\left(\rho_{2}^{2}, p_{2}\right) \in \Gamma_{0}$. Thus

$$
\inf _{\Gamma_{0}} J_{0} \leq J_{0}\left(\rho_{2}^{2}, p_{2}\right)=\inf _{\Gamma^{*}} E_{0},
$$

such that $\inf _{\Gamma^{*}} E_{0}=\inf _{\Gamma_{0}} J_{0}$ and as a consequence,

$$
\left(n_{0}, p_{0}\right):=\left(\rho_{2}^{2}, p_{2}\right)
$$

is a minimizer of $J_{0}$ in $\Gamma_{0}$. Furthermore, if $\left(n_{3}, p_{3}\right) \in \Gamma_{0}$ is a minimizer of $J_{0}$ in $\Gamma_{0}$ then $\left(\sqrt{n_{3}}, p_{3}\right)$ is a minimizer of $E_{0}$ in $\Gamma^{*}$. Hence - since $E_{0}$ has exactly one minimizer in $\Gamma^{*}-\sqrt{n_{3}}=\rho_{2}$ and $p_{3}=p_{2}$ which gives $\left(n_{3}, p_{3}\right)=\left(n_{0}, p_{0}\right)$, i.e. $\left(n_{0}, p_{0}\right)$ is the unique minimizer of $J_{0}$ in $\Gamma_{0}$.

1. Setting $V_{0}=V\left[n_{0}-p_{0}-C\right]$ and $\alpha_{0}:=\alpha_{\delta_{\circ}}, \beta_{0}:=\beta_{\delta_{\circ}}$ (see the end of Step 5), then - due to $\log _{\delta}\left(p_{0}\right)=\log \left(p_{0}\right)$ - the quintuple $\left(n_{0}, p_{0}, V_{0}, \alpha_{0}, \beta_{0}\right)$ is a solution of (8)-(14).

2. The inequalities $0<\delta_{1} \leq p_{0} \leq K_{8}, n_{0} \leq K_{7}^{2}$ and $\left|V_{0}\right| \leq K_{9}$ have already been established in Step 4. It remains to prove that $n_{0}$ is uniformly bounded away from 0 . This follows from a standard truncation argument from (8) employing $\left\|n_{0}\right\|_{L^{\infty}(\Omega)} \leq K_{7}^{2}$ and $\left|V_{0}\right| \leq K_{9}$.

3. has already been proved in Step 4 .

4. We have $p_{0}=\exp \left(V_{0}-\beta_{0}\right)$. Since $V_{0} \in H^{1}(\Omega) \cap L^{\infty}(\Omega)$ we have $p_{0} \in H^{1}(\Omega)$. In analogy, $\sqrt{p_{0}}=\exp \left(\left(V_{0}-\beta_{0}\right) / 2\right) \in H^{1}(\Omega)$. 


\section{References}

[1] R. Adams. Sobolev Spaces. Academic Press, 1975.

[2] M.G. Ancona and G.J. Iafrate. Quantum Correction to the Equation of State of an Electron Gas in a Semiconductor. Phys. Rev. B, 39(13), 9536-9540, 1989 .

[3] N. Ben Abdallah and A. Unterreiter. On the Stationary Quantum DriftDiffusion Model. ZAMP 49, 251-275, 1998.

[4] F. Brezzi and G. Gilardi. Fundamentals of P.D.E for Numerical Analysis. Technical report, Consiglio Nazionale delle Richerche, Corso C. Alberto 5 27100 Pavia - Italy, 1984.

[5] M. Giaquinta. Multiple Integrals in the Calculus of Variations. Annals of Mathematical Studies. Princeton University Press, 1983.

[6] F. Pacard and A. Unterreiter. A Variational Analysis of the Thermal Equilibrium State of Charged Quantum Fluids. Comm PDE 20, 885-900, 1995.

[7] R. Pinnau and A. Unterreiter. The Stationary Current-Voltage Characteristics of the Quantum Drift-Diffusion Model. SINUM 37 (1), 211-245, 1999.

[8] A. Unterreiter. The Thermal Equilibrium Solution of a Generic Bipolar Quantum Hydrodynamic Model. Comm Math Phys 188, 69-88 (1997).

[9] A. Unterreiter The Quantum Zero space Charge Model For Semiconductors. EJAM 10, 395-415 (1999). 